\title{
Synthesis, Properties, and Applications of Multifunctional Magnetic Nanostructures
}

\author{
Vidyadhar Singh, ${ }^{1}$ Rajasekhar Madugundo, ${ }^{2}$ Anil Annadi, ${ }^{3}$ Biswanath Bhoi, ${ }^{4}$ \\ K. Devi Chandrasekhar, ${ }^{5}$ and Murtaza Bohra ${ }^{6}$ \\ ${ }^{1}$ Nanoparticles by Design Unit, Okinawa Institute of Science and Technology (OIST) Graduate University, \\ 1919-1 Tancha, Onna-son, Okinawa 904-0495, Japan \\ ${ }^{2}$ BCMaterials, Bizkaia Science and Technology Park, 48160 Derio, Spain \\ ${ }^{3}$ Institute for Molecular Engineering, University of Chicago, Chicago, IL 60637, USA \\ ${ }^{4}$ Seoul National University, Seoul 08826, Republic of Korea \\ ${ }^{5}$ Department of Physics, National Sun Yat-sen University, Kaohsiung 80424, Taiwan \\ ${ }^{6}$ Mahindra École Centrale College of Engineering, Hyderabad 500043, India \\ Correspondence should be addressed to Vidyadhar Singh; vidya.singh@oist.jp
}

Received 24 July 2017; Accepted 24 July 2017; Published 25 October 2017

Copyright (C) 2017 Vidyadhar Singh et al. This is an open access article distributed under the Creative Commons Attribution License, which permits unrestricted use, distribution, and reproduction in any medium, provided the original work is properly cited.

Magnetic nanostructures have emerged as a novel class of materials being used in variety of applications such as ultra-high-density magnetic recording media, drug delivery, magnetic resonance imaging, sensing, hyperthermia, and catalysts. These magnetic nanostructures can be synthesized in various forms ranging from nanoparticles, nanoclusters, nanorods, nanowires, and nanotubes to the thin films and multilayers, depending upon growth conditions. During last decade, interest in magnetic nanostructures has grown exponentially being inspired by recent advances in nanostructure growth and characterization techniques from which various fascinating properties can be realized which were not observed in the bulk material. These distinct properties can be ascribed to the energies associated with various physical parameters; those are comparable with size of nanostructures. Control over these properties enables researcher to develop new concepts that would lead to novel end products.

In this special issue, total of nine manuscripts were accepted for publication. These manuscripts are mainly focused on synthesis and characterizations of multifunctional magnetic nanostructures and their promising applications. We hope that these articles will give the readers useful information and pathway for futuristic scientific applications. In the following, we will summarize the results from accepted manuscripts.
A. Ehrmann et al. theoretically simulated the magnetic states at remanence for particle of two different shapes at nanoscale. For clusters of these particles, the probability of reaching the additional intermediate onion states in the same field region is strongly reduced with decreased interparticle distance. Significantly, the intermediate onion state, often found in fourfold nanoparticles with open core, which is shown to be stable for interparticle distance down to $12 \mathrm{~nm}$ in specific shape arrangement is of technological interest due to the possibility of using such particles in quaternary memory devices. These findings on understanding the interparticle interactions between both shapes indicate possible solution is in the form of designing new magnetic nanoparticle shapes.

X. G. Li et al. reported a novel method for the analysis of the combined influence of writing head geometry and media properties on writing field performance. They have used the response surface methodology (RSM) for this work. Author analyzed the influence of several parameters such as shielding trailing gap, medium coercivity, and shielding side gap on the effective writing field (EWF) gradient. Their method is useful for evaluation of the bit error rate and SNR of shingled bit patterned recording systems.

W. Zhao et al. studied Cr doped ZnS nanorods synthesized by hydrothermal method. The authors investigated the magnetic and optical properties of $\mathrm{Zn}_{1-x} \mathrm{Cr}_{x} \mathrm{~S}$ nanorods as 
a function of $\mathrm{Cr}$ concentration. The authors found that as the concentration of $\mathrm{Cr}$ increased, the blue emissions intensity improved and the band gap underwent blue shift while the saturation magnetization weakened significantly compared to pure $\mathrm{ZnS}$.

N. A. A. M. Arif et al. proposed a cost effective and simple conventional spin coating method to synthesize high quality sphere-like ZnS:Mn nanocrystalline thin films. The authors investigated the effect of annealing temperature and spin coating speed on the structural and optical properties of Mndoped $\mathrm{ZnS}$ nanocrystalline thin film. The direct band gap for the films was found within the range $4.43 \mathrm{eV}-4.60 \mathrm{eV}$ and the absorption spectra for the films have a limiting wavelength of $250 \mathrm{~nm}$. It was also demonstrated that the decrement in annealing temperature results in improvement of the properties.

I. L. Ardelean et al. developed a facile method to synthesize magnetite nanoparticles with mesoporous structure by coprecipitation method using different stabilizing agents like salicylic acid, glutamic acid, and trichloroacetic acid. They used stabilizing agent to prevent the aggregation of the magnetite nanocrystals and to obtain stable nanostructures even in the biological environment. These nanoparticles are potential candidates for contrast agents as well as targeted carrier for specific diseases, especially cancer.

O. Makarchuk et al. synthesized magnetic nanocomposite sorbents for disposal of synthetic detergents from wastewater. Obtained sorbents based on clay minerals (saponite, palygorskite, and spondyle clay) and magnetite were characterized by X-ray powder diffraction, Mössbauer spectroscopy, and ballistic method with Steinberg magnetometer. The efficiency of magnetic composites and implementing of magnetic separation in adsorption purification was confirmed.

A. Ancira-Cortez et al. described the preparation of a dual-image targeting system composed of magnetic iron oxide nanoparticles functionalized with folic acid and rhodamine in order to achieve and enhance the detection of cancer. Folic acid and rhodamine were conjugated with high efficiency. A significant selectivity and uptake, facilitated by surface modification of iron oxide nanoparticles with folic acid, were demonstrated. The multifunctional system showed suitable physicochemical and biological properties for cell targeting through folate receptors.

Finally, Q. Dai et al. investigated the bacterial magnetosomes (BMs), which have potential in drug delivery vehicles, possessing an iron oxide or iron sulfide core surrounded by a natural lipid membrane shell. They immobilized cytosine arabinoside (Ara-C) effectively on BMs by using various methods such as direct absorption (ABMs), and others include different cross-linkers such as genipin (GP) and glutaraldehyde $(G)$. The results indicate that BMs-based nanoconjugates will potentially find widespread applications in pharmaceutical field. Additionally, H. Xiang et al. prepared $\mathrm{Fe}_{3} \mathrm{O}_{4}$ magnetic nanoparticle coated by PLA and then investigated detail structure, morphology, and biocompatibility of $\mathrm{PLA} / \mathrm{Fe}_{3} \mathrm{O}_{4}$ microsphere. Their study demonstrates that, in comparison with liver cell, liver cancer cells (HepG2) are easy to be disturbed by $\mathrm{PLA} / \mathrm{Fe}_{3} \mathrm{O}_{4}$ nanomagnetic microspheres, which have higher sensitivity and absorption ability. Their finding can take advantage of the susceptible property of cancer cells for carriers to improve targeted drug function.

\section{Acknowledgments}

We would like to express our gratitude to all the authors for their contribution and all reviewers for their critical inputs.

Vidyadhar Singh
Rajasekhar Madugundo
Anil Annadi
Biswanath Bhoi
K. Devi Chandrasekhar
Murtaza Bohra



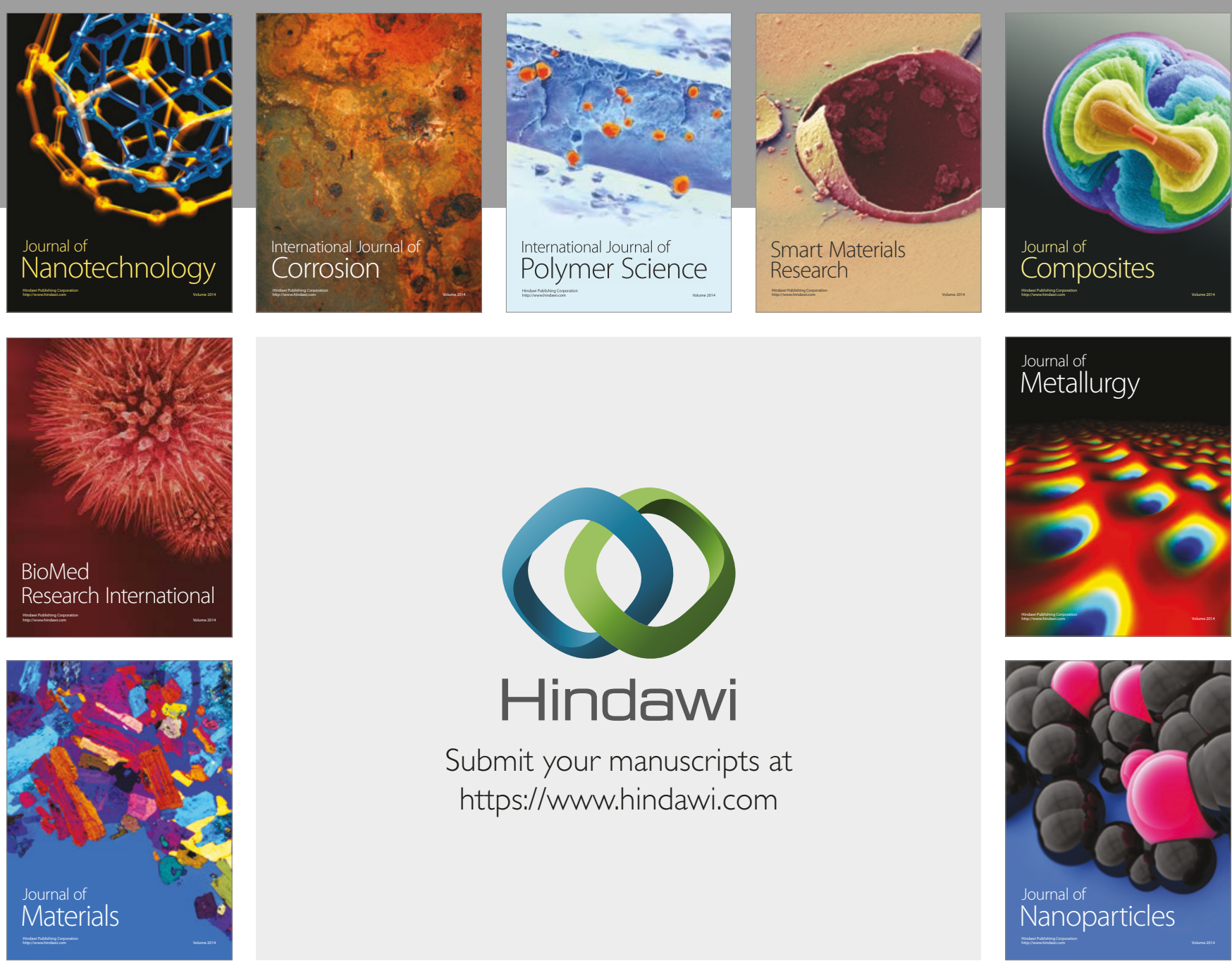

\section{Hindawi}

Submit your manuscripts at

https://www.hindawi.com
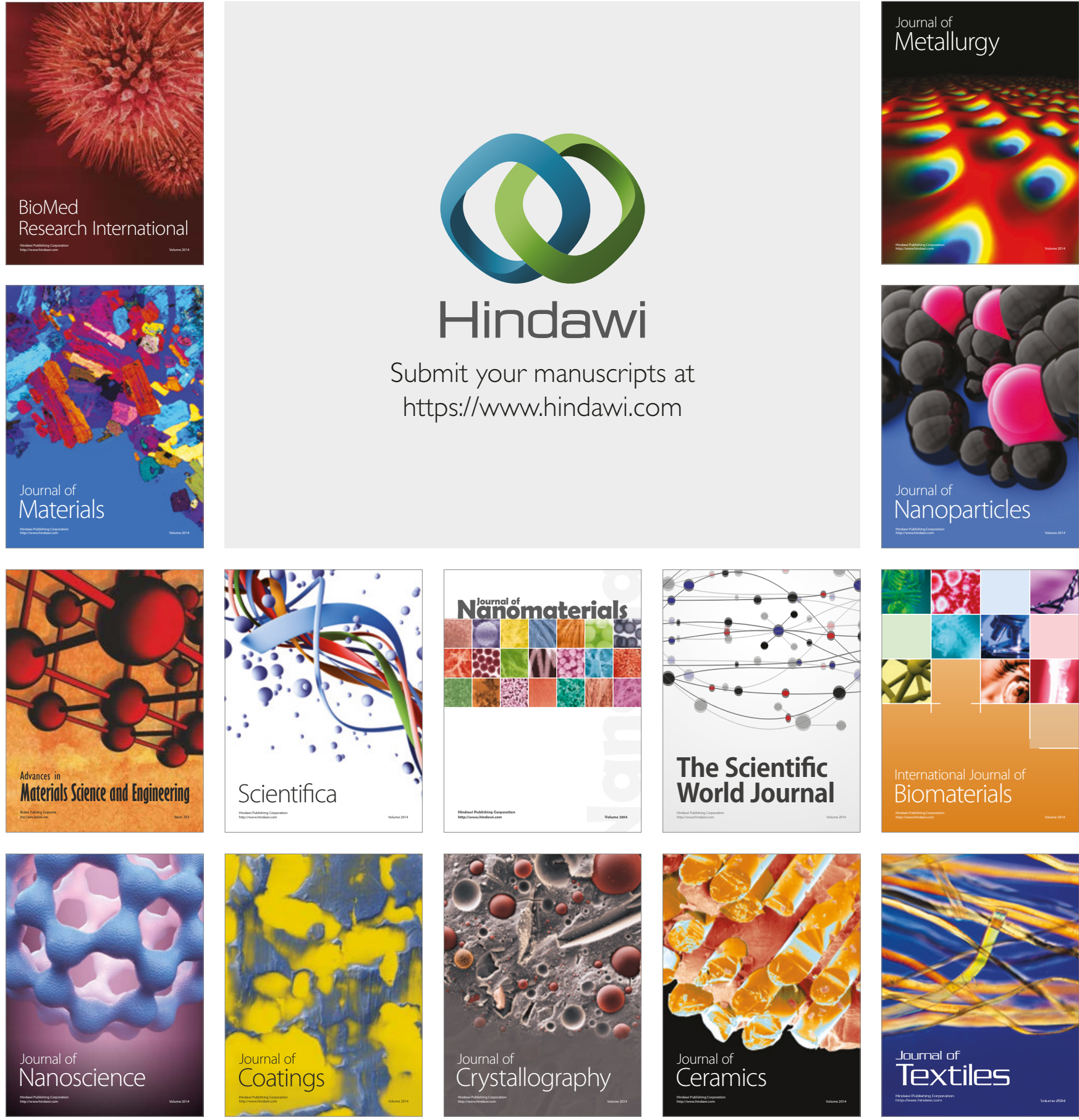

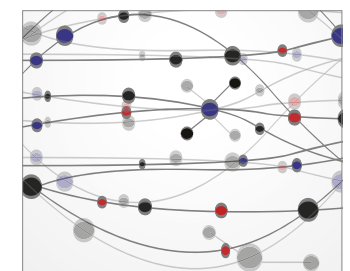

The Scientific World Journal
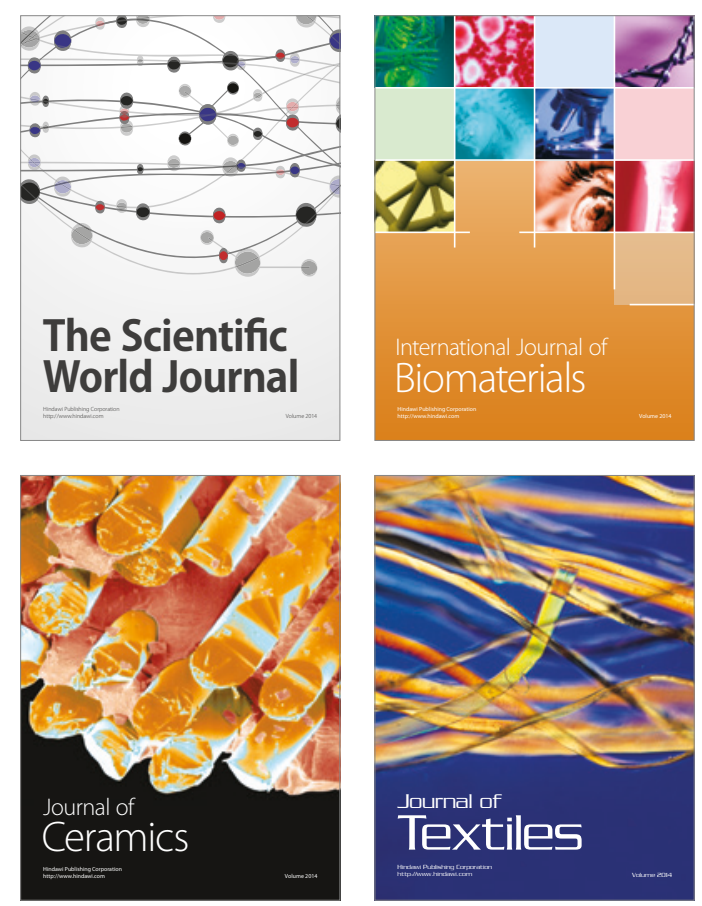